\title{
An Experimental Comparison of Quantum Decision Theoretical Models of Intertemporal Choice for Gain and Loss
}

\author{
Taiki Takahashi $^{1}$, Hiroshi Nishinaka ${ }^{2}$, Takaki Makino ${ }^{2}$, Ruokang Han ${ }^{1}$, Hiroki Fukui ${ }^{2}$ \\ ${ }^{1}$ Department of Behavioral Science, Center for Experimental Research in Social Science, Hokkaido University, Sapporo, Japan \\ ${ }^{2}$ Department of Forensic Psychiatry, National Institute of Mental Health National Center of Neurology and Psychiatry, Tokyo, Japan \\ Email: taikitakahashi@gmail.com
}

Received September 10, 2012; revised October 13, 2012; accepted October 24, 2012

\begin{abstract}
In mathematical physics and psychology, "quantum decision theory" has been proposed to explain anomalies in human decision-making. One of such quantum models has been proposed to explain time inconsistency in human decision over time. In this study, we conducted a behavioral experiment to examine which quantum decision models best account for human intertemporal choice. We observed that a q-exponential model developed in Tsallis' thermodynamics (based on Takahashi's (2005) nonlinear time perception theory) best fit human behavioral data for both gain and loss, among other quantum decision models.
\end{abstract}

Keywords: Discounting; Neuroeconomics; Econophysics; Quantum Decision Theory; Tsallis' Statistics

\section{Introduction}

Intertemporal choice and temporal discounting (devaluetion of delayed reward as delay until its receipt increases) have been attracting attention in quantum decision theory [1], econophysics [2-4], and neuroeconomics [5]. It is to be noted that intertemporal choice has originally been investigated in economics [6]. In these disciplines, several mathematical models have been proposed to account for human intertemporal choice. Notably, [7] proposed intertemporal choice models based on quantum theoreticcal foundations ("quantum decision theory"). In mathematical physics and psychology, recent studies developed this type of quantum decision models in order to explain various anomalies in human decision and cognition [7-9]. However, to date, no study has experimentally examined the explanatory powers of the quantum decision theoretical models for human intertemporal choice. This issue is important for future studies in quantum information and decision theory in mathematical physics, econophysics, and neuroeconomics. In this study, we experimentally examined explanatory powers of intertemporal choice models based on quantum decision theory, in relation to psychophysical theory of intertemporal choice [10] based on Tsallis' thermostatistics [2].

\section{Intertemporal and Probabilistic Choices}

In standard economics, the following exponential time- discount model was proposed [11]:

$$
V(D)=V(0) \exp \left(-k_{e} D\right)
$$

where $V(D)$ is a subjective value of monetary outcome subject receives or pays at delay $D$ and impulsivity parameter $k_{e}$ is an exponential time-discount rate. In behavioral neuroeconomics, a hyperbolic discounting model has been widely employed $[5,6]$ :

$$
V(D)=\frac{V(0)}{1+k_{h} D}
$$

where $V(D)$ is a subjective value of outcome which subject receives/pays at delay $D$ and impulsivity parameter $k_{h}$ is a hyperbolic time-discount rate.

In econophysics, Cajueiro [2] proposed a $q$-exponenttial model based on Tsallis' statistics [12] to generalize exponential and hyperbolic discounting:

$$
V(D)=\frac{V(0)}{\left(1+k_{q}(1-q) D\right)^{1 /(1-q)}}
$$

where $V(D)$ is a subjective value of outcome which subject receives/pays at delay $D$ and impulsivity parameter $k_{q}$ is a $q$-exponential time-discount rate at delay $D=0$. Parameter $q$ generalizes the exponential function. Equation (3) is equivalent to an exponential model Equation (1) when $q$ is 1 and equivalent to a hyperbolic model Equation (2) when $q$ is 0 . When $q$ is less than 1 , the 
agent's temporal discounting is referred to as "decreasing impatience", which may result in preference reversal over time.

Recently, on the other hand, Yukalov and Sornett [1] proposed several types of intertemporal choice models based on quantum decision theory (QDT). In their models, it is basically assumed that

$$
\frac{\mathrm{d}}{\mathrm{d} t}\left|\pi\left(t, t_{0}\right)\right\rangle=-k\left(t, t_{0}\right)\left|\pi\left(t, t_{0}\right)\right\rangle
$$

where $\left|\pi\left(t, t_{0}\right)\right\rangle$ is a "prospect state" and $k\left(t, t_{0}\right)$ is a time-dependent time-decay (discount) rate. It is to be noted that in Yukalov and Sornett's temporal discounting model, temporal discounting is assumed to occur due to a decrease in probability as delay increases, in line with Sozou's evolutionary theory of hyperbolic discounting $[13,14]$. Based on this assumption, Yukalov and Sornett [1] derived several intertemporal choice models (i.e., discount functions, in terms of economic theory). The general form for the Yukalov-Sornett time-discount functions is

$$
\gamma\left(t, t_{0}\right)=\gamma\left(1+\left(t-t_{0}\right)\right)^{-n}
$$

where $\gamma$ and $n$ are free parameters and the time-scale is set at 1 day. [1] further derived several simplified temporal discounting models (Models 1, 2, 2', 3, 4, see [12], for explicit functional forms of the QDT-based models) based on reasonable approximations. However, little is known regarding which model is the best for explaining human intertemporal decision-making. Therefore, we experimentally investigated the explanatory powers of the proposed models based on QDT and the q-exponential time-discount model based on Tsallis' thermostatistics (and Takahashi's logarithmic time perception theory of "hyperbolic" discounting $[10,15,16]$.

\section{Method}

\subsection{Participants}

Forty-one (22 male and 19 female) students were recruited from Chuo University in Japan to take part in the experiment. The mean age was 23.31 years old (standard deviation $=3.20$ ).

\subsection{Procedure}

The participants were asked to perform time discounting tasks of both gain and loss. They were seated individually in a quiet room, facing the experimenter across a table. Then they received a simple instruction that they were asked to choose from a series of alternatives of monetary amounts either gain or loss with certain probabilities and imagine them, though hypothetical, as real money in this experiment. The employed time discount- ing tasks were the same as our previous studies $[15,16]$.

\subsection{Data Analysis}

The procedures of data analysis in the present study is similar to our previous studies $[4,14]$. Switching points of the time discounting tasks were defined as the means of the largest adjusting amount in which the standard alternative choice and the smallest adjusting amount in which the adjusting alternative choice. Indifference points of individuals were calculated by averaging the switching point in the ascending and descending adjusting conditions. The indifference points of the group data were obtained by calculating the medians of individuals' indifference points in order to compare the goodnessof-fit among the models based on QDT and Tsallis' statistics at the group level. The fitness of each equation was estimated with AIC (Akaike Information Criterion) values, which is the most standard criterion for the fitness of mathematical model to observed data. All statistical procedures were conducted with $\mathrm{R}$ statistical language.

\section{Result}

We present fitted parameters and goodness-of-fit (AIC) in Tables 1 (gain) and 2 (loss). From these analyses, we can see that the q-exponential time-discount model fit the behavioral data best for both gain and loss, consistent with logarithmic time-perception theory of intertemporal choice proposed by [10]. It is also to be noted that q vales in the q-exponential discounting were smaller than 1 for both gain and loss, indicating decreasing impatience ("hyperbolic" discounting) for both signed outcomes. Furthermore, we can see that Model 2 and 4 are mathematically equivalent (Tables 1 and 2).

\section{Discussion}

To our knowledge, this study is the first to experimenttally examine time discount models for gain and loss proposed in the Quantum Decision Theory and the q-exponential discounting model based on deformed algebra developed in Tsallis' thermostatistics. Our results demonstrated that 1) Model 2 and 4 (temporal discounting with non-zero limit at large delays) best fit among the quantum decision theoretic intertemporal choice models, and 2) the q-exponential model best fitted human temporal discounting behavior for both gain and loss. It is to be emphasized that, even when novel temporal discounting models proposed in QDT, the q-exponential timediscount model best accounted for human intertemporal choice for both gain and loss, consistent with our previous studies $[10,15,16]$. Therefore, future studies in econophysics, QDT, and neuroeconomics should model 
Table 1. Parameters and AICs of temporal discounting for gain.

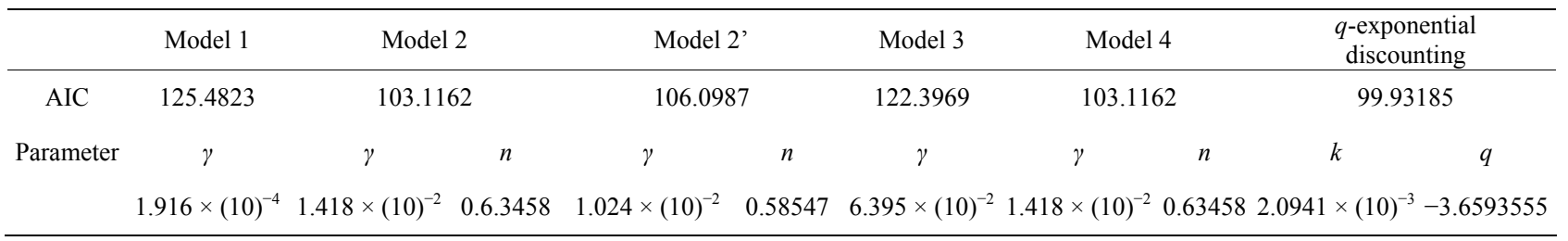

Model 1 is an exponential discounting model, Model 2 reduces to an exponential discounting at short delays, Model 2' is a stretched exponential discounting model, Model 3 is generalized hyperbolic function, Model 4 is close to an exponential model at short delays and tends to non-zero limit at large delays. See Yukalov and Sornette (2009) for details. The q-exponential discounting based on Tsallis' statics is derived from logarithmic time-perception theory of hyperbolic discounting (Takahashi, 2005). Note that smaller AIC indicates better fitting.

Table 2. Parameters and AICs of temporal discounting for loss.

\begin{tabular}{|c|c|c|c|c|c|c|c|c|c|c|}
\hline & Model 1 & \multicolumn{2}{|c|}{ Model 2} & \multicolumn{2}{|c|}{ Model 2' } & Model 3 & \multicolumn{2}{|c|}{ Model 4} & \multicolumn{2}{|c|}{$\begin{array}{c}q \text {-exponential } \\
\text { discounting }\end{array}$} \\
\hline AIC & 105.9509 & \multicolumn{2}{|c|}{99.47295} & \multicolumn{2}{|c|}{99.35718} & 119.1168 & \multicolumn{2}{|c|}{99.47295} & \multicolumn{2}{|c|}{97.9409} \\
\hline \multirow[t]{2}{*}{ Parameter } & $\gamma$ & $\gamma$ & $n$ & $\gamma$ & $n$ & $\gamma$ & $\gamma$ & $n$ & $k$ & $q$ \\
\hline & $5.667 \times(10)^{-5}$ & $8.374 \times(10)^{-4}$ & 0.3502031 & $8.169 \times(10)^{-4}$ & 0.3473363 & 0.024537 & $8.374 \times(10)^{-4}$ & 0.350203 & $1.445 \times(10)^{-4}$ & -2.74 \\
\hline
\end{tabular}

Model 1 is an exponential discounting model, Model 2 reduces to an exponential discounting at short delays, Model 2' is a stretched exponential discounting model, Model 3 is generalized hyperbolic function, Model 4 is close to an exponential model at short delays and tends to non-zero limit at large delays. See Yukalov and Sornette (2009) for details. The $q$-exponential discounting based on Tsallis' statics is derived from logarithmic time-perception theory of hyperbolic discounting (Takahashi, 2005). Note that smaller AIC indicates better fitting.

human intertemporal choice with the $q$-exponential discount model, which can be derived from Takahashi's nonlinear time perception theory of hyperbolic discounting [10].

\section{Acknowledgements}

The research reported in this paper was supported by a grant from the Grant-in-Aid for Scientific Research (Global COE and on Innovative Areas, 23118001; Adolescent Mind \& Self-Regulation) from the Ministry of Education, Culture, Sports, Science and Technology of Japan.

\section{REFERENCES}

[1] V. I. Yukalov and D. Sornette, "Physics of Risk and Uncertainty in Quantum Decision Making," The European Physical Journal B, Vol. 71, No. 4, 2009, pp. 533-548. doi:10.1140/epjb/e2009-00245-9

[2] D. O. Cajueiro, "A Note on the Relevance of the q-Exponential Function in the Context of Intertemporal Choices," Physica A: Statistical Mechanics and Its Applications, Vol. 364, 2006, pp. 385-388.

[3] T. Takahashi, "A Comparison of Intertemporal Choices for Oneself versus Someone Else Based on Tsallis' Statistics," Physica A: Statistical Mechanics and Its Applications, Vol. 385, 2007, pp. 637-644.

[4] T. Takahashi, H. Oono and M. H. Radford, "Empirical Estimation of Consistency Parameter in Intertemporal Choice Based on Tsallis' Statistics," Physica A: Statistical Mechanics and Its Applications, Vol. 381, 2007, pp. 338-342.
[5] T. Takahashi, "Theoretical Frameworks for Neuroeconomics of Intertemporal Choice," Journal of Neuroscience, Psychology, and Economics, Vol. 2, No. 2, 2009, pp. 75-90. doi:10.1037/a0015463

[6] S. Frederick, T. Loewenstein and T. O'Donoghue, "Time Discounting and Time Preference: A Critical Review," Journal of Economic Literature, Vol. 40, No. 2, 2002, pp. 351-401. doi:10.1257/002205102320161311

[7] J. R. Busemeyer, E. M. Pothos, R. Franco and J. Trueblood, "A Quantum Theoretical Explanation for Probability Judgment Errors," Psychological Review, Vol. 118, No. 2, 2011, pp. 193-218. doi:10.1037/a0022542

[8] T.Cheon and T. Takahashi, "Interference and Inequality in Quantum Decision Theory," Physics Letters A, Vol. 375, No. 2, 2010, pp. 100-104. doi:10.1016/i.physleta.2010.10.063

[9] A. Khrennikov, "Ubiquitous Quantum Structure," Springer, Berlin, 2010.

[10] T. Takahashi, "Loss of Self-Control in Intertemporal Choice May Be Attributable to Logarithmic Time-Perception," Medical Hypotheses, Vol. 65, No. 4, 2005, pp. 691-693. doi:10.1016/j.mehy.2005.04.040

[11] P. A. Samuelson, "A Note on Measurement of Utility," The Review of Economic Studies, Vol. 4, 1937, pp. 155161. doi: $10.2307 / 2967612$

[12] C. Tsallis, C. Anteneodo, L. Borland and R. Osorio, "Nonextensive Statistical Mechanics and Economics," Physica A: Statistical Mechanics and Its Applications, Vol. 324, 2003, pp. 89-100.

[13] P. D. Sozou, "On Hyperbolic Discounting and Uncertain Hazard Rates," Proceeding of the Royal Society B Biological Sciences, Vol. 265, 1998, pp. 2015-2020.

[14] T. Takahashi, K. Ikeda and T. Hasegawa, "A Hyperbolic 
Decay of Subjective Probability of Obtaining Delayed Rewards," Behavioral and Brain Functions, Vol. 3, 2007, p. 52. doi:10.1186/1744-9081-3-52

[15] T. Takahashi, H. Oono and M. H. B. Radford, "Psychophysics of Time Perception and Intertemporal Choice Models," Physica A: Statistical Mechanics and Its Appli- cations, Vol. 87, No. 8-9, 2008, pp. 2066-2074.

[16] R. Han and T. Takahashi, "Psychophysics of Time Perception and Valuation in Temporal Discounting of Gain and Loss," Physica A: Statistical Mechanics and Its Applications, Vol. 391, 2012, pp. 6568-6576. 